\title{
Comparative biochemical analysis of wildtype and drug resistant HIV-I integrase subtypes B and C
}

Tamara Bar-Magen ${ }^{* 1}$, Richard D Sloan ${ }^{1}$, Emily I McDonough ${ }^{1,2}$, Verena Faltenbacher ${ }^{1}$, D Aaron Donahue ${ }^{1,2}$, Bjorn D Kuhl ${ }^{1,3}$, Hongtao Xu ${ }^{1}$ and Mark A Wainberg $1,2,3$

Address: ${ }^{1}$ McGill University AIDS Centre, Lady Davis Institute-Jewish General Hospital, Montreal, Quebec, Canada, ${ }^{2}$ Department of Microbiology and Immunology, McGill University, Montreal, Quebec, Canada and ${ }^{3}$ Department of Experimental Medicine, McGill University, Montreal, Quebec, Canada

* Corresponding author

from Frontiers of Retrovirology: Complex retroviruses, retroelements and their hosts

Montpellier, France. 2I-23 September 2009

Published: 24 September 2009

Retrovirology 2009, 6(Suppl 2):P6 doi: 10.1 186/1742-4690-6-S2-P6

This abstract is available from: http://www.retrovirology.com/content/6/S2/P6

(c) 2009 Bar-Magen et al; licensee BioMed Central Ltd.

\section{Objectives}

Subtype specific differences in the development of drug resistance mutations have been reported for reverse transcriptase and protease. Therefore, it is also important to compare the enzymatic activities of purified HIV-1 integrase (IN) from subtypes $\mathrm{B}$ and $\mathrm{C}$ in the wildtype (wt) enzymes, as well as those containing drug resistance mutations. We now report on the enzymatic activities of both wt IN enzymes and those containing either the E92Q or $\mathrm{N} 155 \mathrm{H}$ mutations or both mutations together.

\section{Methods}

To evaluate the enzymatic activities from non-B subtypes we cloned subtype C IN from pINDIE-C1 into a prokaryotic protein expression vector, inserted solubility mutations, described previously for subtype $\mathrm{B}$ IN, and resistance mutations utilizing site-directed mutagenesis. We then expressed and purified the relevant proteins. The catalytic activities of both wt enzymes were analyzed through biochemical assays for 3' processing and strand transfer (3' P and ST respectively). An extensive biochemical characterization of all purified integrase enzymes was performed, both in the presence and absence of IN inhibitors (Raltegravir, Elvitegravir and MK-2048).

\section{Results}

Our results indicate that the enzymatic activities of wt subtype $B$ and $C$ integrases are not significantly different, suggesting that there is little variation between subtypes $\mathrm{B}$ and $C$ in regard to integrase function. In addition, wt enzymes of both subtypes displayed similar sensitivity to the IN inhibitors. The double mutant E92Q/N155H enzymes showed higher resistance to IN inhibitors than did enzymes containing only single mutations, and differences between mutated enzymes of subtype B versus C origin in regard to levels of resistance were demonstrable but not pronounced. This is the first report on the enzymatic activity of both wt and drug resistant subtype C IN enzymes.

\section{Conclusion}

IN from subtypes B and C behave similarly in biochemical assays and in regard to susceptibility to IN inhibitors. 